\title{
Extending the applicability of the four-flux radiative transfer method
}

\author{
Marc A. Gali ${ }^{1}$, Angus R. Gentle ${ }^{1}$, Matthew D. Arnold ${ }^{1}$, and Geoffrey B. Smith ${ }^{1 *}$ \\ ${ }^{1}$ University of Technology Sydney (UTS), 15 Broadway, Ultimo NSW 2007 \\ *Corresponding author: geoff.smith@uts.edu.au \\ Compiled September 29, 2017
}

\begin{abstract}
A generalized four flux method which is capable of modeling and tuning the spectral reflectance of diverse range of complex composite coatings is presented. An example application is exploring and maximising the visible and near IR spectral reflectance available from the diverse structures arising from combinations of the many practical paint ingredients which are available or can be made when applied to different substrates. This requires consideration of scatterers which can differ in composition, particle size, size distribution, fill factor, and are held in place by a variety of organic binders (which typically partially absorb in the near IR). This extended model is further enhanced by an explicit matrix algorithm which allows analysis of diverse multilayer stacks. This is applied to a multilayer and is designed to model useful changes that result from varying pigment fill factor as a function of depth within a layer. A novel feature is the way the scattering impacts on binder or matrix absorptance. The model includes contributions to total absorptance from the scattering pigments and from the binder which can arise in different bands or simultaneously at the same wavelengths. Model accuracy is demonstrated by example results when compared to experimental data on dried single layer paint profiles using imaged cross sections. The model input covering actual pigment and binder properties used are; material, shape, size, and size distributions, mass added, and the measured optical constants from $400 \mathrm{~nm}$ to $2,500 \mathrm{~nm}$ of the un-doped binder resin layer. One interesting novel result is the comparison of a two-layered stack, with bigger particles in the first layer and smaller in the second, to one with the opposite depth profile. () 2017 Optical Society of America
\end{abstract}

OCIS codes: (290.5825) Scattering theory, (290.5850) Scattering, particles, (290.2200) Extinction,(160.2710) Inhomogeneous optical media, (310.6188) Spectral properties

http://dx.doi.org/10.1364/ao.XX.XXXXXX

\section{INTRODUCTION}

Modeling of the reflectance and transmittance of scattering media has been an area of interest for decades, since particle suspensions are ubiquitous in nature (e.g. clouds) and are utilized for practical effect in artificial materials such as coatings and paints [1-4]. The random distribution of the scattering elements makes it impossible to solve these systems analytically; instead Monte Carlo methods are often used requiring a long computational time to obtain accurate results. Also, the radiative transfer problem for diffused light has been presented in the framework of Chandrasekhar's equation [5] solving the problem for different incident angles [6-9] which give accurate solutions for diffused light. On the other hand, methods based on flux balance equations usually present a much faster way to obtain results [10-12]; two-flux models assume fully diffused fluxes propagating in opposite directions, among those there is the well known Kubelka-Munk theory [13, 14], which has been intensively used and researched [15-17]. In particular, the four-flux method has been used broadly for its easy applicability and the good balance that it offers between complexity and accuracy [10, 18-20], consequently several developments have been made in its formulation and applicability [21-23]; nevertheless implementations presented to this point still have a number of limitations.

In this paper diluted dispersions are considered. As a consequence the four-flux method is better suited to study them than two-flux methods, due to the importance of both collimated and diffused light. The four-flux offers versatility in modeling the dependence between collimated and diffused fluxes as the coating thickness.

The main innovations presented in this work are:

1. the capability to analyze the impact of absorbing matrices,

2. an implementation with an admix of different kinds of scatterers including different particle sizes and particle compo- 
sitions,

3. a systematic way to implement particle size distributions,

4. a clear and specific methodology to determine model parameters,

5. a convenient matrix formalism to apply the generalized four flux method developed in this paper in a multilayer system.

This paper is structured in the following way: in section 2 we will present the problem of study with the classical FourFlux balance equations and define the classical balance equation using effective volumetric parameters, which will allow us to generalize its applicability; the solutions of our method will be presented in a normalized way in order to avoid possible indeterminations; in section 3 the effective volume coefficients will be presented; in section 4 we will introduce the formalism to include particle size distribution into the model; in section 5 we present a matrix formalism to analyze multi-layered stacks; in section 6 we will present example results, discuss the influence that different factors have in the total reflectance and transmittance and test the models ability to fit measured optical response and structural parameters of two different paint layers; in section 7 we will compare simulated with experimental results.

\section{FOUR-FLUX MODEL}

Throughout this paper we will consider the problem represented schematically in Figure 1. A slab of thickness $Z$ that separates two media has incident and exit fluxes on each side made up of two collimated beams and two diffuse fluxes. In our model we will assume the same hypotheses as the classical papers [10, 21], which is that collimated light can become diffused but not the other way around: this is not a very restrictive assumption, since the probability that diffused light becomes collimated due to scattering of random distributed particles is negligible; it also assumes an infinite slab in the $x, y$-axis, and consequently reflectance and transmittance on these directions are omitted. These hypotheses should be taken into account depending on which scenario is modeled, since the edge effect sometimes has great importance. In our research we want to model paints. As a consequence the difference in magnitude between the incident direction (z-axis) length scale of microns; and the paint plane dimensions ( $x, y$-axis), scale of centimeters or meters, make the four flux hypothesis applicable.

Thus, the balance equations will depend on the difference of the refractive index between each interface, the scattering appearing inside the slab due to the added particles, and any absorption caused by the particles and the matrix itself. The diffuse scattering profile depends on a parameter called forward average path length $(\epsilon)$, which weights the amount of scattered light entering a solid angle. The value of $\epsilon$ is a measure of the impact of particle spatial distribution on average path length. If distributed isotropically hence randomly the particles will lead to isotropic optical response. If distributed anisotropically with greater linear density along some directions than others, anisotropic scattering results. Vargas [24] discussed this parameter and analytical ways to define it. In this work we assume isotropic diffuse scattering, which yields a value of $\epsilon=2$.

The differential equations in the normal direction $\mathrm{z}$ are written in terms of the forward (J) and backward (I) fluxes, divided into collimated (subscript c) and diffuse (subscript d) components:

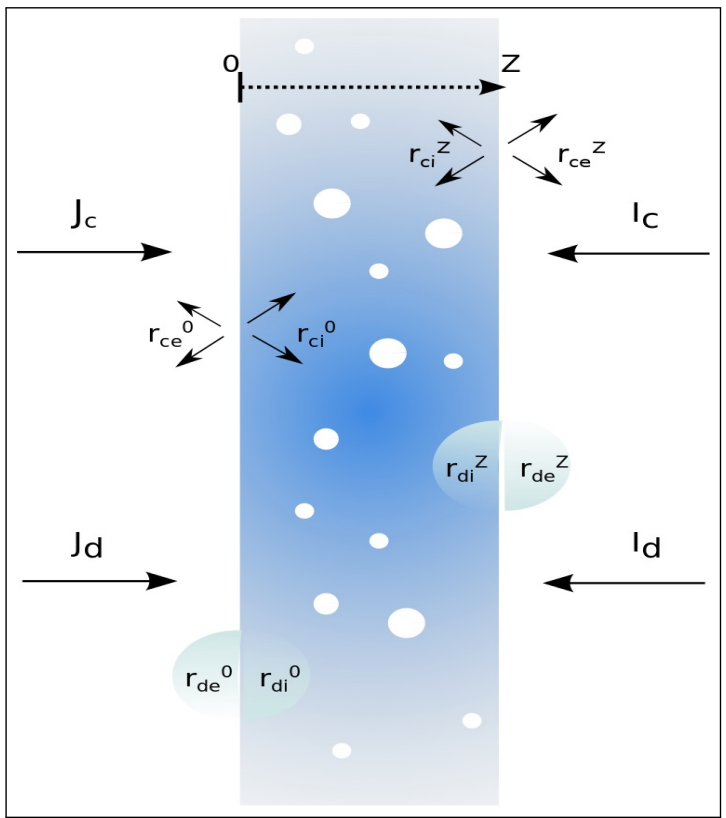

Fig. 1. Diagram of the four fluxes and interface parameters used in the four flux model. $\mathrm{J}_{c, d}, \mathrm{I}_{c, d}$ are the incident collimated and diffused fluxes at each side of the slab; $\mathrm{Z}$ is the slab thickness; $\mathrm{r}_{i}^{j}$ are the reflection coefficients at the interfaces; superscript 0 refers to the side where $\mathrm{J}$ fluxes enter the slab, while $\mathrm{Z}$ refers to the side where I fluxes are incident; subscript $d$ refers to diffused light, c for collimated; while subscript i refers to the interior edge and e to the exterior edge.

$$
\begin{aligned}
\frac{d I_{c}}{d z} & =\left(K_{c}+S\right) I_{c}, \\
\frac{d J_{c}}{d z} & =-\left(K_{c}+S\right) J_{c}, \\
\frac{d I_{d}}{d z} & =-\tilde{\zeta} I_{c}-\tilde{\beta} J_{c}+\epsilon\left(K_{d}+\tilde{\beta}\right) I_{d}-\epsilon \tilde{\beta} J_{d}, \\
\frac{J_{d}}{d z} & =\tilde{\beta} I_{c}+\tilde{\zeta} J_{c}+\epsilon \tilde{\beta} I_{d}-\epsilon\left(K_{d}+\tilde{\beta}\right) J_{d},
\end{aligned}
$$

where $\tilde{\zeta}, \tilde{\beta}$ are the averaged forward and back scattered light per unit length, $K_{c, d}$ is the total absorption per unit of length of collimated (c) or diffused (d) light, and $S$ the total scattered light per unit of length. In the classical four flux models, those parameters are defined as the scattering and absorption rates. In most studies to date the particles were embedded in a non-absorbing matrix. However in our model these rates, hence the various coefficients, have to be modified since Mie scattering rates are now influenced by the extinction occurring in the matrix while absorption rates involve the sum of two extinction processes, matrix and particle absorption. In the next section we will give a detailed explanation of those coefficients.

\section{A. Total reflection and transmission coefficients}

The solution of a position dependent differential equation is usually given by the expression that defines the variable (here each J or I) in terms of its position within the slab e.g. J(z) for any $\mathrm{z}$ between 0 and $\mathrm{Z}$. In the case of the four flux problem, we are mainly interested in the light fluxes and electromagnetic energy travelling away from and entering the slab. These can be 
expressed in terms of reflection and transmission coefficients at each side of the slab and these total $\mathrm{R}$ and $\mathrm{T}$ values at $z=0$ and $z=Z$ can be broken down into collimated or diffused radiation if desired. For example we use three subscripts to distinguish each $\mathrm{R}$ and $\mathrm{T}$ physical component. These are for (i) incident collimated to exit collimated (cc) (ii) incident collimated to exit diffuse (cd) (iii) incident diffuse to exit diffuse (dd). Superscripts $0(z=0)$ and $Z(z=Z)$ denote the different boundaries and in this work boundary 0 for total reflectance $R^{0}$ is our main technical interest but we will also look at $T^{Z}$ since some transmittance data has been obtained on free-standing composites. Hence we derive expressions that are equivalent to those in reference [25], but it is important to notice that our expressions are simplified in order to avoid non-physical numerical singularities that previous expressions could present. This presents a more robust framework to accurately study the extreme cases were the thickness of the slab becomes large or tends to zero, or the cases where the extinction coefficient becomes very large. For example, these expressions have allowed us to compute results for composites with both small size parameter and thick matrices. The $\mathrm{R}$ and $\mathrm{T}$ coefficients for collimated, diffused, and diffused from collimated light at both ends of the slab $(\mathrm{z}=0$ and $\mathrm{z}=\mathrm{Z})$ are:

$$
\begin{aligned}
& R_{c c}^{0}=r_{c}^{0}+\frac{r_{c}^{Z}\left(1-r_{c}^{0}\right)^{2}}{\varphi \tilde{D_{1}}}, \\
& R_{c d}^{0}=\frac{\left(1-r_{d i}^{0}\right)\left(1-r_{c}^{0}\right)}{\tilde{D_{1}}{\tilde{D_{2}}}_{2}}\left[\tilde{C_{1}}+\tilde{C_{2}}+\tilde{C_{3}}\right], \\
& R_{d d}^{0}=r_{d e}^{0}+\frac{\left(1-r_{d 1}^{0}\right)\left(1-r_{d e}^{0}\right)}{\tilde{D}_{2}} \tilde{C}_{4}, \\
& T_{c c}=\frac{\left(1-r_{c}^{0}\right)\left(1-r_{c}^{Z}\right)}{D_{1}}, \\
& T_{c d}^{0}=\frac{\left(1-r_{d i}^{Z}\right)\left(1-r_{c}^{0}\right)}{\tilde{D_{1}} \tilde{D_{2}}}\left(\tilde{B_{1}}+\tilde{B_{2}}+\tilde{B_{3}}\right), \\
& T_{d d}^{0}=\frac{\left(1-r_{d i}^{Z}\right)\left(1-r_{d e}^{0}\right)}{D_{2}}\left(1-\alpha_{3}^{2}\right),
\end{aligned}
$$

$C_{i}, B_{i}, D_{i}$ coefficients are defined in the appendix (sec.10), the $r_{c}^{0}, r_{d i}^{0}, r_{d e}^{0}$ are the reflection coefficients at the $z=0$ interface of collimated light, diffused light inside the matrix and diffused light outside the matrix, respectively; equivalently the coefficients $r_{i}^{Z}$ are the respective reflection coefficients at the interface $z=Z$. These coefficients are obtained using Fresnel equations, and in order to obtain the diffused reflections we average the Fresnel reflection coefficients over the hemisphere.

Finally, the problem is solved using the previous equations (5-10) to express the total reflectance and transmittance at both ends of the slab.

$$
\begin{aligned}
R^{0} & =\frac{\left(R_{c c}^{0}+R_{c d}^{0}\right) J_{c}+R_{d d}^{0} J_{d}}{J_{c}+J_{d}}, \\
T^{Z} & =\frac{\left(T_{c c}^{0}+T_{c d}^{0}\right) J_{c}+T_{d d}^{0} J_{d}}{J_{c}+J_{d}}, \\
R^{Z} & =\frac{\left(R_{c c}^{Z}+R_{c d}^{Z}\right) I_{c}+R_{d d}^{Z} I_{d}}{I_{c}+I_{d}}, \\
T^{0} & =\frac{\left(T_{c c}^{Z}+T_{c d}^{Z}\right) I_{c}+T_{d d}^{Z} I_{d}}{I_{c}+I_{d}} .
\end{aligned}
$$

\section{EFFECTIVE VOLUME COEFFICIENTS}

In this section we will introduce the effective volume coefficients, which will allow us to include in the energy balance equations characteristic of the Four-Flux method the effects of different kind of particles, same particles but with different sizes, or the absorption of the matrix. These effects are crucial when we try to model real matrices, which we will show in the discussion section. The balance equations that define our problem study the change of flux per unit of length, i.e. $d I=I d z$, which means that if we want to use a coefficient to express loss or gain of this flux, it has to have units of [length $]^{-1}$, this is one of the main things that we will have to have in mind when defining the effective volume coefficients.

\section{A. Absorption and scattering coefficients}

In this work we assume that the scatterers inside the medium are spherical and at a low concentration. Thus, under these conditions, Mie theory can be used to obtain the scattered and absorbed energy by the particles. Also, as the series expansion used in Mie Theory can present convergence problems for large size parameters or very absorbing media, in those cases the scattering results have been validated using Debye series formulation [26-28]. As has been done in the classical paper [10, 21] and as explained before, we have to normalize dimensions of length for cross sections, i.e. the cross sections from Mie theory have units of inverse of area ([length $]^{-2}$ ), in other words they express the intensity absorbed or scattered per surface. In order to obtain a parameter with the proper dimensions it seems natural to use the number density of this kind of scatterers. In doing so and assuming that the particles are symmetrical we can estimate the energy absorbed or scattered per unit of length. Thus, the absorption (scattering) parameter will be defined as:

$$
\begin{aligned}
k_{a b s}^{i} & =\eta_{i} \cdot C_{a b s}, \\
s^{i} & =\eta_{i} \cdot C_{s c a t},
\end{aligned}
$$

$\eta_{i}$ is the number density of the $\mathrm{i}^{\text {th }}$ scatterer.

On the other hand, if we want to obtain the absorption due to the matrix, first we should obtain the absorption coefficient of the medium for collimated and diffused light, i.e. we want to obtain how much light is absorbed only by the matrix when the collimated and diffused light are traveling in the considered system. Thus, as the traveled distance of the diffused light depends on the average path-length, $\epsilon$ should be considered when studying the effective decay of diffused light in the system. To do so we will consider an equivalent dispersive medium (diffused light has same average path-length as the original system), only composed of the particular matrix, therefore in this scenario the balance equations 1-4 are reduced to the following:

$$
\begin{aligned}
& \frac{d J_{c}}{d z}=-K_{c} J_{c}, \\
& \frac{d J_{d}}{d z}=-\epsilon K_{d} J_{d} .
\end{aligned}
$$

These differential equations are straightforward to solve and have as a solution a decaying exponential:

$$
J_{i}=J_{0, i} \cdot \exp \left[-\alpha_{i}\right],
$$

where $i=c, d$ and $\alpha_{c}=K, \alpha_{d}=\epsilon K$. Thus, using previous expressions we can obtain the skin depth $\left(\delta_{S}\right)$ in terms of the 
decaying constant $\left(\alpha_{i}\right)$, which allows us to find the following relation [29]:

$$
\alpha_{i}=\frac{2}{\delta_{s}}=\frac{4 \pi n_{2}}{\lambda},
$$

where $n=n_{1}+i n_{2}$, is the refractive index, being $n_{2}$ the extinction coefficient. Then, we can define the absorption collimated and diffused coefficients due to the matrix:

$$
\begin{aligned}
& k_{c}=\frac{4 \pi n_{2}}{\lambda}, \\
& k_{d}=\frac{4 \pi n_{2}}{\lambda \cdot \epsilon} .
\end{aligned}
$$

Therefore, we can define a characteristic coefficient of the composite that expresses the total energy loss due to absorption per unit of length. These effective coefficients $\left(K_{c(d)}\right)$ will be the result of doing a volume average of the absorption coefficients of each element $\left(k_{i}\right)$. Thus, using previous definitions we can write a discrete expression for the effective coefficient, considering the volume occupied for each element yields:

$$
K_{c(d)}=\sum_{i}^{N} k_{a b s}^{i}+k_{c(d)} \cdot\left(1-\sum_{i}^{N} f_{i}\right),
$$

where $f_{i}$ is the fill factor of particles of type $i$ of $N$ different types.

Notice that the previous expression converges to the case of a non-absorbing matrix for $\alpha=0$ which of course, by construction, also must agree with the case of a bulk absorbing material for the case $N=0$, when the absorption is exactly the absorption coefficient, i.e. $K=\alpha$.

Similarly as we did for the loss due to absorption, we can define a characteristic parameter of the slab that expresses the scattering occurring inside the slab per unit of length. In this situation we only need to consider the particles inside the slab, since they are responsible for this scattering.

$$
S=\sum_{i}^{N} s^{i}
$$

\section{A.1. Forward and back scattering ratios}

In the four-flux model, an additional two coefficients called the forward and back scattering coefficients are required. They are not to be confused with the forward and back scattering of the particles, instead these coefficients are a ratio that indicates how much of the scattered light from the particle goes towards the forward or back hemisphere. Thus, as in this work we are assuming spherical particles, these coefficients can be defined using Mie theory. Using notation of Bohren-Huffman [30], the scattered light on the two main polarizations depends on the coefficients $S_{1}, S_{2}$, defined as follows:

$$
\begin{aligned}
& S_{1}=\sum_{n} \frac{2 n+1}{n(n+1)}\left(a_{n} \pi_{n}+b_{n} \tau_{n}\right), \\
& S_{2}=\sum_{n} \frac{2 n+1}{n(n+1)}\left(a_{n} \tau_{n}+b_{n} \pi_{n}\right),
\end{aligned}
$$

where $\tau_{n}$ and $\pi_{n}$ are the angular dependent functions, thus if we want to know the ratio of scattered light in the forward or back hemisphere we have to integrate over the respective angles and then average the polarizations.

$$
\begin{aligned}
& \zeta_{1,2}=\frac{\int_{0}^{2 \pi} \int_{0}^{\pi / 2}\left|S_{1,2}\right|^{2}|\cos \theta| d \Omega}{\int_{0}^{2 \pi} \int_{0}^{\pi}\left|S_{1,2}\right|^{2}|\cos \theta| d \Omega}, \\
& \beta_{1,2}=\frac{\int_{0}^{2 \pi} \int_{\pi / 2}^{\pi}\left|S_{1,2}\right|^{2}|\cos \theta| d \Omega}{\int_{0}^{2 \pi} \int_{0}^{\pi}\left|S_{1,2}\right|^{2}|\cos \theta| d \Omega},
\end{aligned}
$$

where $d \Omega=\sin \theta d \theta d \phi$ is the solid angle, $\left|S_{1,2}\right|^{2}$ are the scattered irradiance per unit incident irradiance given that the incident polarization is perpendicular $\left(\left|S_{1}\right|^{2}\right)$ or parallel $\left(\left|S_{2}\right|^{2}\right)$ to the scattering plane given by $\theta$. Note that the cosine term should be considered, because $\zeta$ and $\beta$ are the ratios of scattered light in the forward and backward directions, so the projection on that direction is what should be considered. The reason to use the absolute value of the cosine is that these ratios consider the amount of energy scattered in each projection and to do so the sign of the cosine should be omitted, since the direction information is implicit on $\zeta$ and $\beta$.

Finally averaging over the two polarizations,

$$
\begin{aligned}
& \zeta=\frac{1}{2}\left(\zeta_{1}+\zeta_{2}\right), \\
& \beta=\frac{1}{2}\left(\beta_{1}+\beta_{2}\right),
\end{aligned}
$$

and by definition they satisfy the relation $\zeta+\beta=1$.

Therefore, we can define the amount of light scattered into the forward or backward hemisphere for each kind of scatterer in order to obtain the final forward and back scattering ratios used in the balance equations 1-4. Finally in order to obtain the average scattered light in the forward and back hemisphere, we will use the ratios that we just defined (eq.29-30), and use them to average the scattered light.

$$
\begin{aligned}
& \tilde{\zeta}=\sum_{i}^{N} \zeta^{i} \cdot s^{i}, \\
& \tilde{\beta}=\sum_{i}^{N} \beta^{i} \cdot s^{i},
\end{aligned}
$$

where $\beta^{i}, \zeta^{i}$ are the coefficients of the $\mathrm{i}^{\text {th }}$ scatterer, and $s^{i}$ its total scattered light.

\section{SCATTERER SIZE DISTRIBUTION}

In previous works this problem has been usually solved considering only one kind of scatterer with a fixed size, but in a real sample what we really have are particles with a range of different sizes. Thus, accounting for this diversity of scatterers we can improve the realism of this model, and hence obtain a broadened spectrum as seen in experiments.

As has been shown in the previous section, using effective coefficients we can define the effect of scatterers with different Mie characteristics. In this section we will apply the same principle but for scatterers of the same composition, but different size. Thus, we will define the parameters of a particular kind of particles using the distribution function of their radius to weight the total fill factor of that kind of particles.

$$
k=\int_{r_{0}-\Delta}^{r_{0}+\Delta} \eta(r) \cdot C(r) d r
$$


where $C(r)$ is the cross-section of the particle of size $r$ and $\eta(r)$ is the probability density function of particle radii, which is given by the number of particles with size $r$ per unit of volume (units of $\mathrm{m}^{-3}$ ). Therefore, if we know how the sizes are statistically distributed, we can obtain the effective coefficient of all of them combined.

From a computational point of view it is more suitable to define the discrete version of previous equation as a finite sum:

$$
k \sim \sum_{i=-M}^{M} \eta\left(r_{i}\right) \cdot C\left(r_{i}\right),
$$

with $2 M+1$ being the number of points that discretize the distribution, thus for $M \rightarrow \infty$ the sum converges to the integral (equation 33).

\section{MULTILAYER FORMALISM}

In this section an iterative matrix algorithm is presented to solve a multilayer problem using the four-flux method in each slab. A four flux multilayer method has been studied before [25], nevertheless in that article the propagation phase matrix was not explicitly done, only continuous equations where presented. Therefore the aim of this section is to present explicitly the matrix formalism for an arbitrary number of layers (a similar approach was used by Simonot et al [31], also at the time of writing another multilayer 4 flux approach was published [32]).

Let us assume a system of slabs as presented in diagram figure 2, where the number of layers increases in the $\mathrm{z}$-axis direction; interfaces are labelled as follows: increasing along the $z$ direction, the first interface of each layer is labelled with 0 and the ending interface with $\mathrm{Z}$. In order to find the propagation matrix from one layer to another, we need to know the incoming and outgoing fluxes of a particular slab.

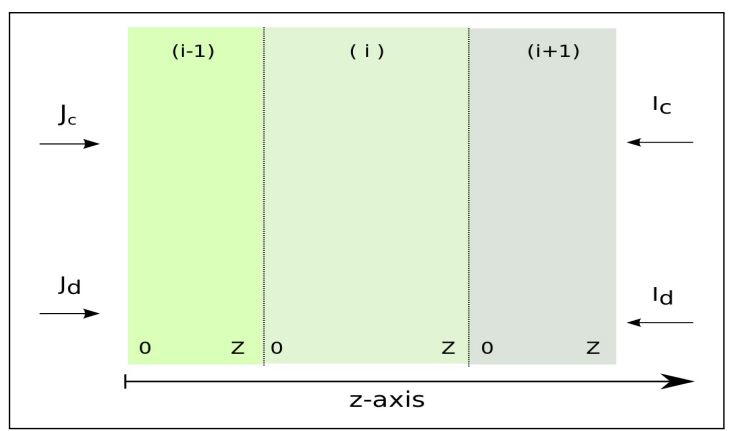

Fig. 2. Diagram of the multilayer structure and notation.

$$
\begin{aligned}
I_{c}^{Z}(i-1) & =I_{c}^{0}(i+1) T_{c c}(i)+J_{c}^{Z}(i-1) R_{c c}^{0}(i), \\
I_{d}^{Z}(i-1) & =I_{c}^{0}(i+1) T_{c d}^{Z}(i)+I_{d}^{0}(i+1) T_{d d}^{Z}(i) \\
& +J_{c}^{Z}(i-1) R_{c d}^{0}(i)+J_{d}^{Z}(i-1) R_{d d}^{0}(i), \\
J_{c}^{0}(i+1) & =J_{c}^{Z}(i-1) T_{c c}^{0}(i)+I_{c}^{0}(i+1) R_{c c}^{Z}(i), \\
J_{d}^{0}(i+1) & =J_{c}^{Z}(i-1) T_{c d}^{0}(i)+J_{d}^{Z}(i-1) T_{d d}^{0}(i) \\
& +I_{c}^{0}(i+1) R_{c d}^{Z}(i)+I_{d}^{0}(i+1) R_{d d}^{Z}(i),
\end{aligned}
$$

where the brackets with the $\mathrm{i}$ arguments denote the layer.

$$
\left\{\begin{aligned}
I_{c}^{0}(i+1) & =I_{c}^{Z}(i), & & J_{c}^{0}(i+1)=J_{c}^{Z}(i), \\
I_{d}^{0}(i+1) & =I_{d}^{Z}(i), & & J_{d}^{0}(i+1)=J_{d}^{Z}(i) .
\end{aligned}\right.
$$

Using equations (35-38) and imposing the conservation of the flux between interfaces eq.(39), we can obtain a linear system that relates two adjacent slabs, which can be expressed in matrix form eq.(40). In order to obtain explicitly the flux-propagation matrix, it is more suitable to write a matrix equation relating the fluxes at both interfaces of a particular layer.

$$
\begin{aligned}
& \left(\begin{array}{cccc}
T_{\mathcal{c}} c & 0 & 0 & 0 \\
T_{\mathcal{c}} d^{0} & T_{d} d^{0} & 0 & 0 \\
-R_{\mathcal{c}} c^{0} & 0 & 1 & 0 \\
R_{\mathcal{c}} d^{0} & R_{d} d^{0} & 0 & -1
\end{array}\right)\left(\begin{array}{c}
J_{\mathcal{c}}(i-1) \\
J_{d}(i-1) \\
I_{\mathcal{c}}(i-1) \\
I_{d}(i-1)
\end{array}\right)= \\
& \left(\begin{array}{cccc}
1 & 0 & -R_{\mathcal{c}} c^{Z} & 0 \\
0 & 1 & -R_{c} d^{Z} & -R_{d} d^{Z} \\
0 & 0 & T_{c} c^{Z} & 0 \\
0 & 0 & -T_{c} d^{Z} & -T_{d} d^{Z}
\end{array}\right)\left(\begin{array}{c}
J_{c}(i) \\
J_{d}(i) \\
I_{c}(i) \\
\left.I_{d}(i)\right)
\end{array}\right)
\end{aligned}
$$

where all reflectance and transmittance coefficients are the coefficients of the $\mathrm{i}^{\text {th }}$ layer. Expressing previous equation in matrix form we can generalize the flux transfer for an arbitrary number of layers.

$$
A \cdot F_{(i-1)}=B \cdot F_{(i)}
$$

with $F_{i}=\left(J_{\mathcal{c}}(i), J_{d}(i), I_{\mathcal{C}}(i), I_{d}(i)\right)^{T}$, thus we can easily express the transfer matrix from eq.41.

$$
\begin{aligned}
& F_{(i-1)}=A_{i}^{-1} B_{i} F_{(i)}, \\
& F_{(i-1)}=M_{i} F_{(i)} .
\end{aligned}
$$

By induction it is easy to see that we can write the flux $F$ at the first interface of the stack in terms of the flux at the final interface of it.

$$
\begin{gathered}
F_{0}=\phi \cdot F_{\tau}, \\
\phi=\prod_{i=1}^{L} M_{i},
\end{gathered}
$$

where $\prod_{i=1}^{L} M_{i}=M_{1} \cdot M_{2} \cdot M_{3} \cdots M_{L}$. Finally, the last step to do is to relate the incident flux (initial condition) with the outgoing flux of the whole stack. The incident fluxes are $X_{0}=\left(J_{c}(0), J_{d}(0), I_{\mathcal{C}}(\tau), I_{d}(\tau)\right)^{T}$, and the outgoing fluxes are $X=\left(J_{c}(\tau), J_{d}(\tau), I_{c}(0), I_{d}(0)\right)^{T}$, with $\tau$ the total stack thickness.

\section{RESULTS AND DISCUSSION}

One of the main purposes of this work is to present the overall effect in a turbid medium caused by different kind of scatterers: different in composition, size and size dispersion. It is important to know the extent that exploiting these differences and allowing their combinations in one sample will allow wider degrees of spectral tuning of the optical response. Thus, in order to compare such effects we will first consider a fixed layer thickness of $380 \mu \mathrm{m}$. It is important to clarify that we are fixing this thickness parameter in order to emphasize the relevance of other key parameters; nonetheless, if the objective was to analyse or improve a particular system the thickness of the composite should 
also be considered, especially if the matrix is absorbing. Also, if not stated otherwise we are considering size distributions with standard deviation of $20 \%$ of the mean radius.

We have implemented this formalism using MATLAB; some of the Mie scattering routines where based on C. Mätzler code [33].

\section{A. Binder absorption}

The aim of this section is to convince the reader of the effectiveness and value of the modifications presented in this work. With this goal in mind we will present systematic simulations that show, first the effect of these effective coefficients and second, the improvement of the accuracy that they bring. Thus, we will be able to show the improvement in the agreement with experimental data due to the incorporation of the binder absorption by comparing results obtained without binder absorption but all other model parameters retained.
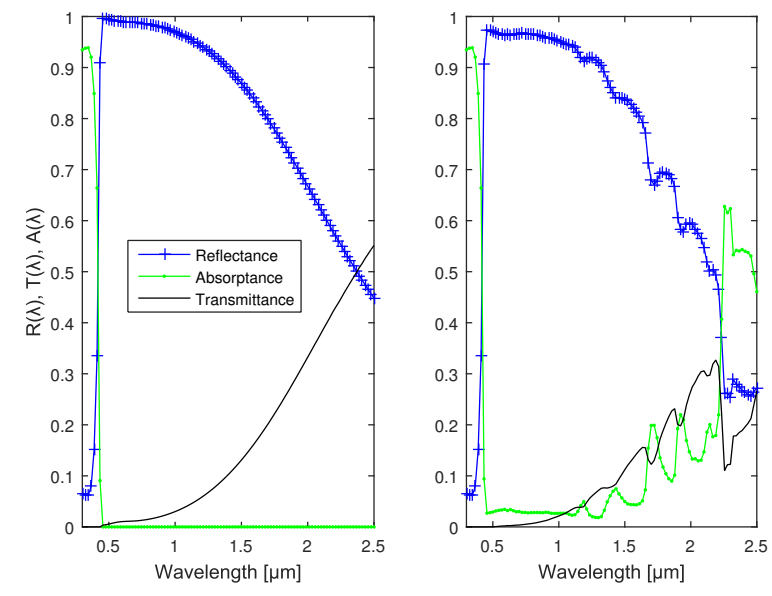

Fig. 3. Simulations of the spectra of acrylic paint with a normal distribution of $\mathrm{TiO}_{2}$ particles of mean radius $0.08 \mu \mathrm{m}$ and fill factor $10 \%$; (a) without binder absorption; and (b) with absorption of the actual Acrylic resin used included.

Figure 3 shows that the effect of simulating the binder absorption is obvious, with strong absorption bands evident in the infrared region. From figure 4 it can be easily seen that the difference in reflection in the IR is due to added acrylic polymer related absorption (green lines). We can also appreciate that in the visible-NIR the binder adds significant absorptance and therefore the reflectance profile in this region also changes considerably. For instance, if we were interested in improving the reflectance in the visible we might consider adding smaller particles in the mixture in order to add backscattered light in this particular region. Also, it is interesting to see the high absorption in the ultraviolet region. In figure 4 this band appears in both simulations, the one with and without binder absorption. This fact tells us that this absorption comes from the embedded particles, indeed this absorption region matches closely with the inter-band absorption edge of rutile $\mathrm{TiO}_{2}$.

The study of the NIR absorption bands resulting from the binder is a crucial matter in coatings used for cooling, therefore being able to model this effect accurately can help us to improve coatings, so there is less absorption in the IR.

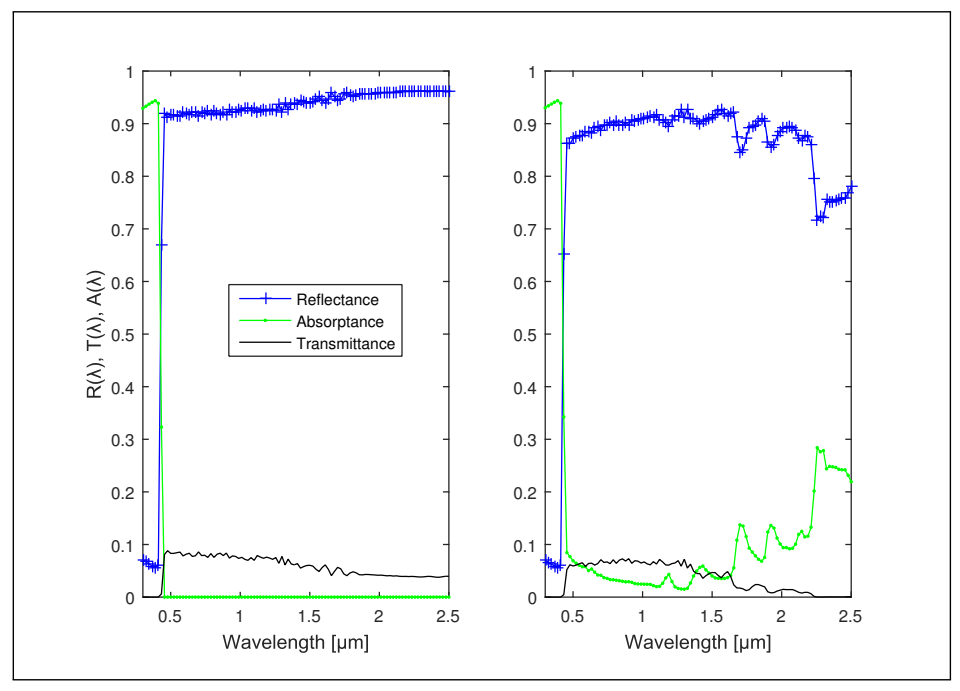

Fig. 4. On the left side we can see a non-absorbing acrylic paint with a normal distribution of $\mathrm{TiO}_{2}$ particles of mean radius $1 \mu \mathrm{m}$ and fill factor $10 \%$. Right graph shows acrylic paint with a normal distribution of $\mathrm{TiO}_{2}$ particles of mean radius $1 \mu m$ and fill factor $10 \%$.

\section{B. Distribution sizes}

In Figure 5 we show the reflectance of acrylic paint for different size of scatterers and solar energy wavelengths. It can be seen that particles with radius between $0.3-0.4 \mu \mathrm{m}$ yield the highest average reflectance over the whole wavelength spectrum, while these smaller particles are also the ones that yield reflectance values of nearly $100 \%$ across the visible range. This is because they backscatter more in this wavelength region. On the other hand bigger particles reflect and backscatter more strongly in the NIR.

By comparing different composites in which mean particle diameter $\mathrm{d}$ changes in large steps over a wide range, with its standard deviation $\sigma$ a fixed fraction of $d$, and particle volume fraction fixed we can systematically study the way configuration changes can be used to increase transmission or reflection of light in particular wavelength bands. As an example we consider the case of a white acrylic based paint with $\mathrm{TiO}_{2}$ pigments as scatterers. Thus, in Figure 6 we present the reflectance of four different paint compositions, where we fix the volume fraction of scatterers, but vary their mean diameter $d$ while setting the standard deviation at $0.1 d$ about each $d$. It is interesting to see that the back-scattering ratio, which is defined as the fraction of light scattered into the half-hemisphere in the backward direction, is strongly dependent on the size as can be seen in the bottom-left image on Figure 6. In this picture we can see that smaller particles are more efficient at backscattering overall, but this advantage diminishes at NIR wavelengths as their scattering there is less than at visible wavelengths. In contrast, bigger particles become much more efficient at backscattering at these longer wavelengths. Nevertheless, what matters is the product of backscattered ratio to total scattered light, in other words, how much scattered intensity goes into the backward hemisphere. Thus, even though for wavelengths around $2 \mu \mathrm{m}$ small particles scatter more in the forward direction, the integrated scattering rate of smaller particles is large. Integrated solar reflectance clearly decreases as diameter increases since the solar intensity falls beyond $1 \mu \mathrm{m}$. This analysis links the total 
spectral reflectance presented on the top-left image in figure 6 to the directional composition of internal scattering. On the other hand, if we were more interested in improving transmittance, we should consider bigger particles, since they back-scatter less on average across the whole solar wavelength spectra, as can be seen in the black lines on the two bottom images on figure 6 .

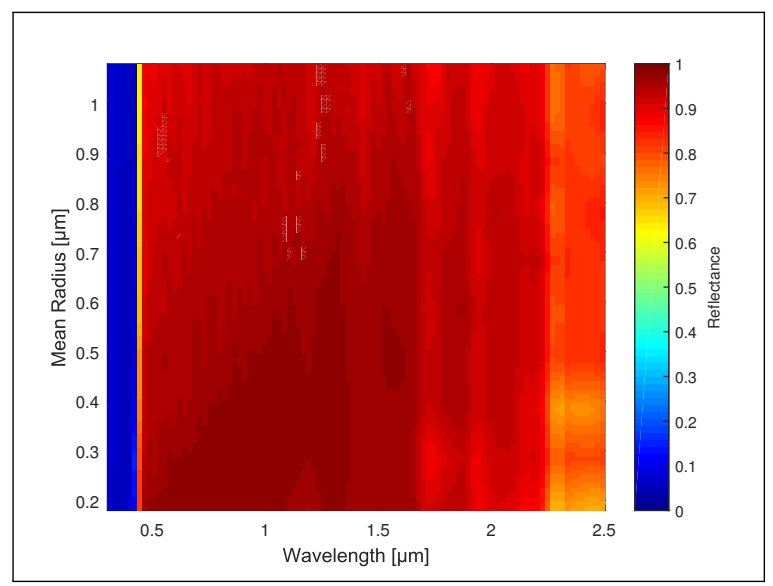

Fig. 5. Shows the reflectance for different scatterer sizes and wavelengths.

If spectral adjustment of backscattering to total scattering ratio is of interest a response that combines those of small and big particles can help. In order to achieve that we have two main options: the first would be choosing a dominant size, the mean size, and to increase the standard deviation of the size distribution about a single $d$ value, which will yield a broader spectral response. On the other hand, two or more considerably different mean $d$ values can provide a different spectral response, so then we may have to include two or more different distributions of diameter $d$ which may be separate or partially overlap as a function of $d$. Figure 7 follows the first idea of one mean $\mathrm{d}$ value. There it can be seen that the wider the distribution is, the more the different spectral responses come together. As the Gaussian approaches a delta function, the spectral response approaches that for a composite with mono-disperse, single diameter particles.

On the other hand, sometimes we will want the response of two distinct distributions, and in those cases, we will follow the second approach. As an example of this influence in figure 8 we compare three types of composites, two of them are characterized by a single size distribution of scatterers with well separated mean diameter values of $0.2 \mu \mathrm{m}$ and $3 \mu \mathrm{m}$, whereas the third example is a result of the weighted combination of the two separate spreads. It can be clearly seen that in doing so a composite is achieved that maintains the very high visible reflectance of the small particles, and the high NIR reflectance achievable with big particles. In the bottom images of figure 8 it can be appreciated that the mixed composite has backscattering and total scattering spectral responses in between the values arising with either a small or large mean radius. Thus it seems intuitive to think that by mixing two particle supplies with very different mean diameters an improvement of overall solar reflectance will occur in the final composite.

These examples shown that mixing different size particles with tailored size distributions can be used to adjust spectral diffuse response over a wide range. This revised 4-flux model is an excellent and flexible composite layer design tool as demon-

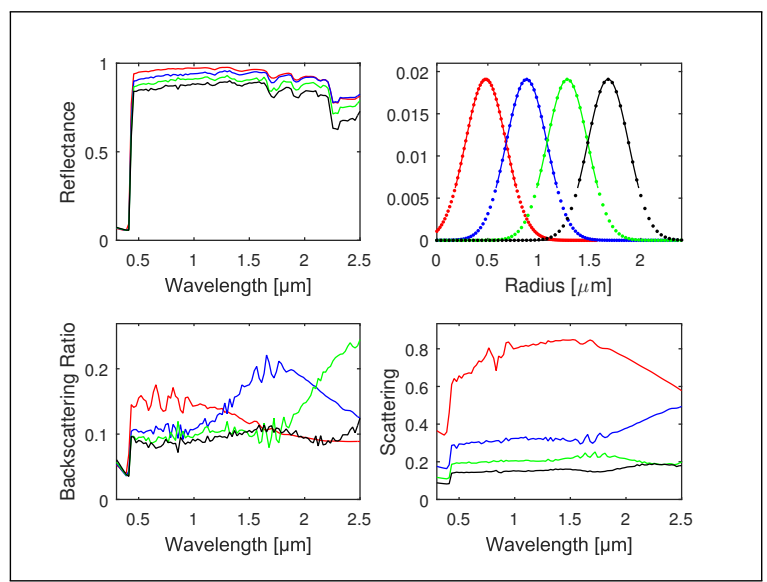

Fig. 6. (Top-Left) Reflection of acrylic paint for different size particle distributions. (Top-Right) Gaussian distribution of particle radius for each paint. (Bottom-Left) Back-scattering ratio of each distribution. (Bottom-Right) Total scattering of each distribution.

strated by its ability to optimize the hemispherical reflectance spectrum of heterogeneous composites, by taking advantage of the spectral variations in their radiative-transfer profiles which are very sensitive to particle size.

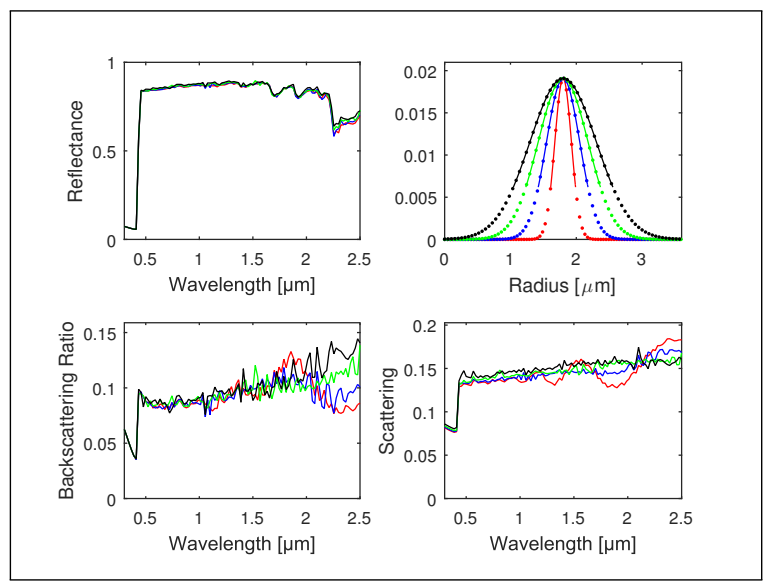

Fig. 7. Shows the effect of varying the standard deviation of the composite size distribution. This system represents an acrylic based paint with $14 \%$ of $\mathrm{TiO}_{2}$ particles with fixed mean radius of $1.8 \mu \mathrm{m}$.

\section{Analysis of a two-layer stack}

Very often natural or artificial composites present a grading in composition, which can be modelled as a multi-layered structure. For instance, some heterogeneous paint solutions that dry into composites with a gradient in composition, which can change the overall optical response. The aim of this subsection is to show the capability of the formalism introduced in section 5, for different configurations. In particular, we will study a two layer stack, where both layers have same binder, same volume ratio of composites and same thickness, but different scatterer size.

Figures 9 shows that there is a significant difference in the optical response due to the arrangement of particles. In figure 9(a), where the first layer contains the big particles, the reflectance 

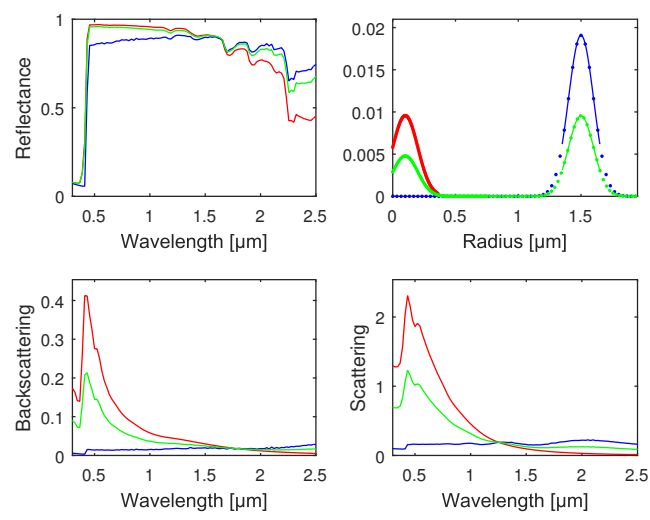

Fig. 8. Top-left figure shows the reflectance of 3 different paints. Top-right graph shows the size distribution of each paint. Bottom-left shows the backscattering ratio of each paint. Bottom-right shows the total scattering of each composition.

spectra is relatively flat, and importantly the absorption in the NIR is much lower than for the opposite configuration (fig 9(b)). This result can be explained considering previous results presented in this paper; bigger particles backscatter more at long wavelengths corresponding to the bigger absorption bands. Thus, reflecting those wavelengths as soon as they get inside the stack is an easy way to prevent absorption.

\section{VALIDATING RESULTS WITH EXPERIMENTAL DATA}

Finally, our model is tested by its ability to model experimental data using structural information obtained from two samples whose spectral hemispherical reflectance and transmittance had been measured. Including binder absorption and particle (e.g. paint pigment) size distributions significantly improved the agreement obtained between experimental and model data. The experimental hemispherical reflectance data was collected using a Perkin Elmer Lambda 950 with 150mm integrating sphere. The refractive index of the binder was determined via preparation of a binder-only sample on a silicon wafer measured via ellipsometry, using a JA Woollam V-VASE ellipsometer, combined with spectroscopic transmittance of the same free-standing film after peeling it off the silicon wafer substrate. The $\mathrm{TiO}_{2}$ refractive index data was modeled using a Tauc-Lorentz oscillator model matching the bandgap to the experimental data.

\section{A. Sample preparation}

Two samples were made following the same process: mixing the compounds and leaving the samples to dry for one week, after which the weights of the composites were estimated. Paint used in figure 10 was made mixing water $(0.53 \mathrm{~g})$, acrylic $(2.25 \mathrm{~g})$ and $\mathrm{TiO}_{2}$ (0.74g small size, $0.1 \mathrm{~g}$ big size); after drying the volatile component loss leaves weights of: $1.24 \mathrm{~g}$ acrylic, small size $\mathrm{TiO}_{2}$ $0.41 \mathrm{~g}$ and big size $\mathrm{TiO}_{2} 0.06 \mathrm{~g}$; and a final thickness of $380 \mu \mathrm{m}$. Using the final weight of the compounds the fill factors of $\mathrm{TiO}_{2}$ particles were found to be $7.72 \%$ for the small size, and $1.79 \%$ for the big size. Paint represented by figure 11 was made mixing water $(0.67 \mathrm{~g})$, acrylic $(1.46 \mathrm{~g})$ and $\mathrm{TiO}_{2}(0.40 \mathrm{~g})$; after the drying process the compound weights got reduced to: acrylic $(0.5 \mathrm{~g})$ and $\mathrm{TiO}_{2}(0.34 \mathrm{~g})$. The estimated fill factor for the $\mathrm{TiO}_{2}$ was $15 \%$. (a)
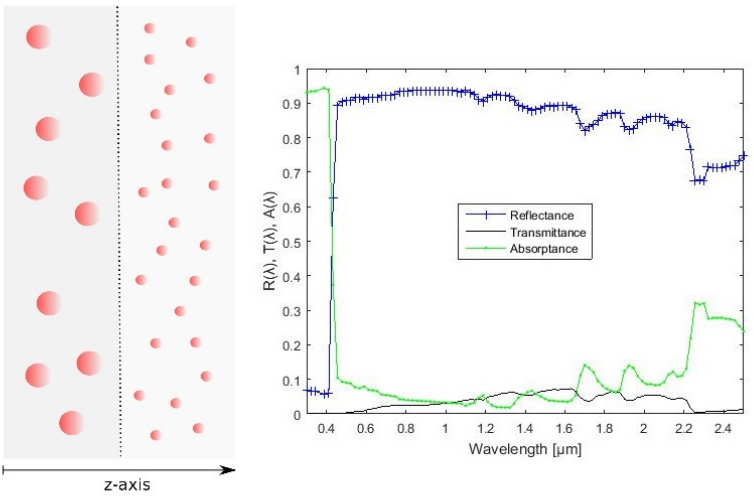

(b)

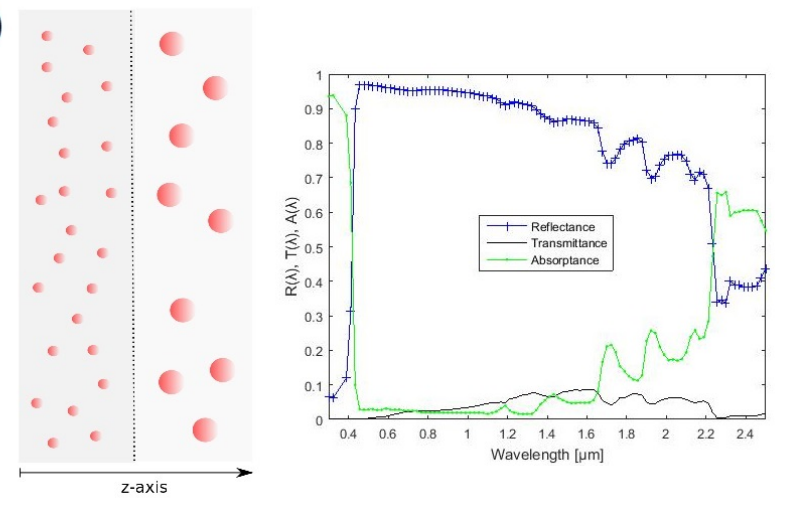

Fig. 9. (a) Left image is a cartoon of the system modeled in the right image. The system is a two-layered stack with acrylic matrix and a $7 \%$ of $\mathrm{TiO}_{2}$ scatterers for both layers; first layer, particles with mean radius $1 \mu \mathrm{m}$ and standard distribution $200 \mathrm{~nm}$; second layer, particles with mean radius $100 \mathrm{~nm}$ and standard distribution of $20 \mathrm{~nm}$. Reflectance, transmittance and absorptance are calculated for incidence fluxes from the left. (b) Left image is a cartoon of the system modeled in the right image. The system is a two-layered stack with acrylic matrix and a $7 \%$ of $\mathrm{TiO}_{2}$ scatterers for both layers; first layer, particles with mean radius $100 \mathrm{~nm}$ and standard distribution of $20 \mathrm{~nm}$; second layer, particles with mean radius $1 \mu \mathrm{m}$ and standard distribution 200nm. 


\section{B. Comparing results}

In order to fit simulation with experimental results, the scatterers size and fill factor have been used as a fitting parameter, thus the best fitting results give a close estimate of the average size of the pigments and their fill factor.

Figure 10 compares the experimental and simulated data. The position of the absorption bands of the binder allows a good match to the experimental optical result over most of the solar spectral range. Reflectance agrees with some minor quantitative differences in the depths of the absorption bands. In order to obtain this result we used two distribution sizes, one with mean radius of $10 \mathrm{~nm}$ and the other with mean radius $180 \mathrm{~nm}$. In both cases the distributions follow a Gaussian with standard deviation set at $10 \%$ of the mean diameter value. Thus, combining different distribution allowed us to match the experimental behavior in both the visible and IR. However, it is important to test the response of other distributions to determine if this result is unique. This is because as shown in the previous sections the reflectance of a composite is very sensitive to the size and volume fraction of the scatterers inside, therefore different combinations of them could give a similar result. This is why we cannot use only the reflectance to fit optical data. Some of the variables must be constrained to avoid any ambiguity on structural impacts. For instance, the total volume fraction of scatterers can be measured, or the size distribution of the composites can be found using microscopy. In addition, pigment supplies often come with information on mean pigment diameters and their distribution which can also be used. Figures 10 and 11 show that the simulated results agree with the experimental data at all wavelengths apart from details in some NIR absorption bands. Differences between simulation and experiment could be attributable to differences between the refractive index data of the acrylic used in the simulation which was separately characterized for index, and its actual refractive index in the measured material after formulation and drying, for example some voids might be present. We also note that we have not considered multiple-scattering which could affect the reflectance spectra.

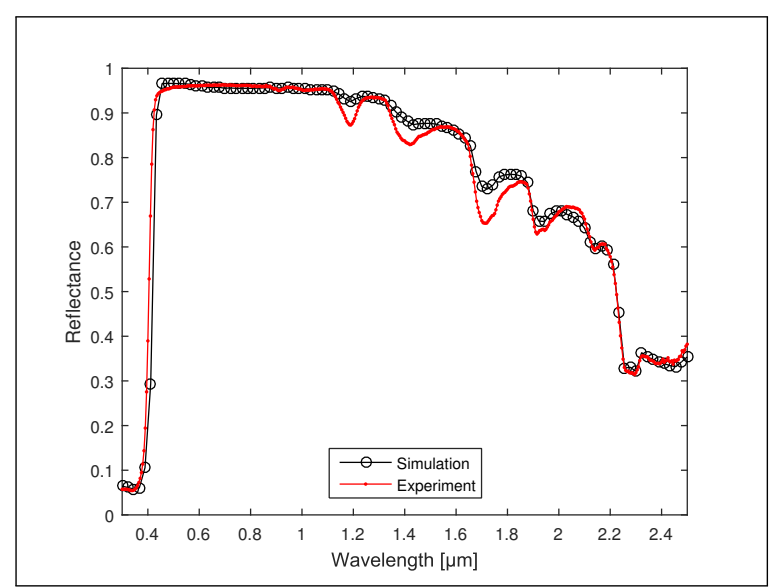

Fig. 10. Comparison between the reflectance of experimental and simulated data of an acrylic paint with $6 \% \mathrm{TiO}_{2}$ scatterers where their size follows a normal distribution with mean radius $\mu_{0}=90 \mathrm{~nm}$ and standard deviation $\sigma=18 \mathrm{~nm}$, and a $1 \%$ $\mathrm{TiO}_{2}$ with a size distribution characterized by a mean radius $\mu_{0}=500 \mathrm{~nm}$ and standard deviation of $\sigma=100 \mathrm{~nm}$.

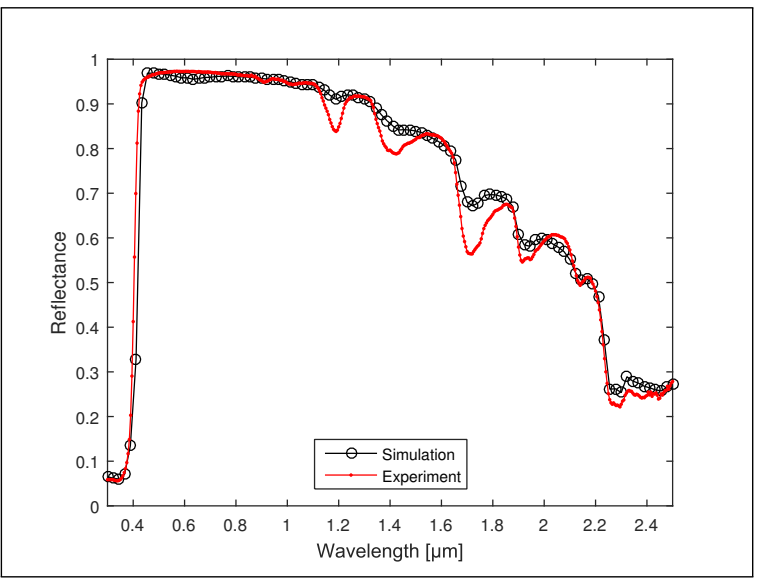

Fig. 11. Comparison between the reflectance of experimental and simulated data of an acrylic paint with $14 \% \mathrm{TiO}_{2}$ scatterers where their size follows a normal distribution with mean radius $\mu_{0}=80 \mathrm{~nm}$ and standard deviation $\sigma=16 \mathrm{~nm}$.

\section{CONCLUSION}

In this paper we introduced an extended version of the classical four-flux method, explained how to obtain all the model coefficients needed and then how to analyse heterogeneous media with different composites. Including any attenuation in the matrix is important. Using this method we can easily analyze the extreme cases where material thickness becomes very large, or when extinction governs the flux transfer. However it is important to note that in our formalism we are assuming non-dense media. For the study of a highly packed medium multi-scattering effects should be considered [18, 34, 35].

Finally, good agreement was obtained between experimental reflectance and reflectance obtained using our model with structural data obtained independently using a SEM. In a future publication we will use this method to optimize white paints in order to improve cooling of buildings exposed to solar radiation.

\section{FUNDING INFORMATION}

The authors acknowledge financial support from the Australian Research Council Discovery grant DP14010200 and the Australian Government Research Training Program Scholarship (RTP).

\section{APPENDIX}

The coefficients used in section 2.A are defined here.

$$
\begin{aligned}
& \tilde{C_{1}}=e^{-2 \sqrt{A_{1}} Z}\left(\alpha_{3}-r_{d i}^{Z}\right)\left[r_{c}^{Z} e^{-2 \Gamma Z}\left(\alpha_{2}-\alpha_{1} \alpha_{3}\right)+\left(\alpha_{1}-\alpha_{2} \alpha_{3}\right)\right] \\
& \tilde{C_{2}}=\left(1-\alpha_{3} r_{d i}^{Z}\right)\left[r_{c}^{Z} e^{-2 \Gamma Z}\left(\alpha_{1}-\alpha_{2} \alpha_{3}\right)+\left(\alpha_{2}-\alpha_{1} \alpha_{3}\right)\right] \\
& \tilde{C_{3}}=\phi^{-1} \cdot\left(1-\alpha_{3}^{2}\right)\left[r_{c}^{Z}\left(r_{d i}^{Z} \alpha_{2}-\alpha_{1}\right)+\left(r_{d i}^{Z} \alpha_{1}-\alpha_{2}\right)\right] \\
& \tilde{C_{4}}=\alpha_{3}\left(1-\alpha_{3} r_{d i}^{Z}\right)-e^{-2 \sqrt{A_{1}} Z}\left(\alpha_{3}-r_{d i}^{Z}\right) \\
& \tilde{D_{1}}=1-r_{c}^{Z} r_{c}^{0} e^{-2 \Gamma Z} \\
& \tilde{D_{2}}=\left(1-\alpha_{3} r_{d i}^{Z}\right)\left(1-\alpha_{3} r_{d i}^{0}\right)-e^{-2 \sqrt{A_{1}} Z}\left(\alpha_{3}-r_{d i}^{Z}\right)\left(\alpha_{3}-r_{d i}^{0}\right)
\end{aligned}
$$




$$
\begin{aligned}
& \tilde{B_{1}}=e^{-\left(\Gamma+2 \sqrt{A_{1}}\right) Z}\left(\alpha_{3}-r_{d i}^{0}\right)\left[r_{c}^{Z}\left(\alpha_{1}-\alpha_{2} \alpha_{3}\right)+\left(\alpha_{2}-\alpha_{1} \alpha_{3}\right)\right], \\
& \tilde{B_{2}}=e^{-\Gamma Z}\left(1-\alpha_{3} r_{d i}^{0}\right)\left[r_{c}^{Z}\left(\alpha_{2}-\alpha_{1} \alpha_{3}\right)+\left(\alpha_{1}-\alpha_{2} \alpha_{3}\right)\right], \\
& \tilde{B_{3}}=e^{-\sqrt{A_{1}} Z}\left(1-\alpha_{3}^{2}\right)\left[e^{-2 \Gamma Z} r_{c}^{Z}\left(r_{d i}^{0} \alpha_{1}-\alpha_{2}\right)+\left(r_{d i}^{0} \alpha_{2}-\alpha_{1}\right)\right] .
\end{aligned}
$$

where $\Gamma=K_{e f f}+S_{e f f}$ is the extinction parameter, $\varphi \equiv e^{\Gamma Z}$ and the relation between the coefficients with and without tilde depends on an exponential:

$$
\begin{aligned}
\phi & =\varphi \cdot \psi=e^{\left(\Gamma+\sqrt{A_{1}}\right) Z}, \\
C_{i} & =\phi \cdot \tilde{C}_{i} \quad(i=1,2,3), \\
C_{4} & =\psi \cdot \tilde{C_{4}} \\
D_{1} D_{2} & =\varphi \tilde{D_{1}} \cdot \psi \tilde{D_{2}}=\phi \cdot \tilde{D_{1}} \tilde{D_{2}} .
\end{aligned}
$$

Coefficients $A_{1}, \alpha_{i}$ are those introduced by Tonon et al [25].

\section{REFERENCES}

1. G. B. Smith and C. G. Granqvist, Green Nanotechnology: Solutions for Sustainability and Energy in the Built Environment (CRC Press, Boca Raton, USA, 2010).

2. H. Gonome, M. Baneshi, J. Okajima, A. Komiya, and S. Maruyama, "Controlling the radiative properties of cool black-color coatings pigmented with $\mathrm{CuO}$ submicron particles," Journal of Quantitative Spectroscopy and Radiative Transfer 132, 90-98 (2014).

3. J. Chai, Q. Cheng, M. Si, Y. Su, Y. Zhou, and J. Song, "Numerical simulation of white double-layer coating with different submicron particles on the spectral reflectance," Journal of Quantitative Spectroscopy and Radiative Transfer 189, 176-180 (2017).

4. M. Baneshi, H. Gonome, A. Komiya, and S. Maruyama, "The effect of particles size distribution on aesthetic and thermal performances of polydisperse TiO 2 pigmented coatings: Comparison between numerical and experimental results," Journal of Quantitative Spectroscopy and Radiative Transfer 113, 594-606 (2012).

5. S. Chandrasekhar, Radiative Transfer (Dover, New York, 1960).

6. C. Siewert, "A concise and accurate solution to Chandrasekhar's basic problem in radiative transfer," Journal of Quantitative Spectroscopy and Radiative Transfer 64, 109-130 (2000).

7. S. K. Barman, "The solution of the radiative transfer equation for polarized light by the Chandrasekhar' s discrete ordinate method," 66, 509-517 (2000).

8. M. Elias and G. Elias, "New and fast calculation for incoherent multiple scattering." Journal of the Optical Society of America A. 19, 894-901 (2002).

9. M. Elias and G. Elias, "Radiative transfer in inhomogeneous stratified scattering media with use of the auxiliary function method." Journal of the Optical Society of America. A 21, 580-9 (2004).

10. B. Maheu, J. N. Letoulouzan, and G. Gouesbet, "Four-flux models to solve the scatering transfer equation in terms of Lorenz-Mie parameters," Applied Optics 23, 3353-3362 (1984).

11. M. Hébert and J.-M. Becker, "Correspondence between continuous and discrete two-flux models for reflectance and transmittance of diffusing layers," Journal of Optics A: Pure and Applied Optics 10, 1-6 (2008).

12. L. A. Dombrovsky, J. Randrianalisoa, and D. Baillis, "Modified two-flux approximation for identification of radiative properties of absorbing and scattering media from directional-hemispherical measurements." Journal of the Optical Society of America 23, 91-8 (2006).

13. P. Kubelka, "New Contributions to the Optics of Intensely LightScattering Materials. Part I," Journal of the Optical Society of America 38, 448 (1948).
14. P. Kubelka, "New Contributions to the Optics of Intensely LightScattering Materials. Part II: Nonhomogeneous Layers," Journal of the Optical Society of America 44, 330 (1954).

15. L. Fukshansky and N. Kazarinova, "Extension of the Kubelka-Munk theory of light propagation in intensely scattering materials to fluorescent media," Journal of the Optical Society of America 70, 1101-1111 (1980).

16. W. E. Vargas and G. A. Niklasson, "Applicability conditions of the Kubelka-Munk theory," Applied Optics 36, 5580 (1997).

17. R. Molenaar, J. J. Bosch, and J. R. Zijp, "Determination of Kubelka-Munk scattering and absorption coefficients by diffuse illumination," Applied Optics 38, 2068-2077 (1999).

18. W. E. Vargas, "Optimization of the diffuse reflectance of pigmented coatings taking into account multiple scattering," Journal of Applied Physics 88, 4079 (2000).

19. W. Vargas and G. Niklasson, "Pigment mass density and refractive index determination from optical measurements," Journal of Physics Condensed Matter 9, 1661-1670 (1997).

20. K. Laaksonen, S. Y. Li, S. R. Puisto, N. K. J. Rostedt, T. Ala-Nissila, C. G. Granqvist, R. M. Nieminen, and G. A. Niklasson, "Nanoparticles of TiO2 and VO2 in dielectric media: Conditions for low optical scattering, and comparison between effective medium and four-flux theories," Solar Energy Materials and Solar Cells 130, 132-137 (2014).

21. W. E. Vargas, "Generalized Four-Flux Radiative Transfer Model," Applied Optics 37, 2615 (1998).

22. C. Rozé, T. Girasole, G. Grehan, G. Gouesbet, and B. Maheu, "Average crossing parameter and forward scattering ratio values in four-flux model for multiple scattering media," Optics Communications 194, 251263 (2001).

23. C. Rozé, T. Girasole, and A. G. Tafforin, "Multilayer four-flux model of scattering, emitting and absorbing media," Atmospheric Environment 35, 5125-5130 (2001).

24. W. E. Vargas and G. A. Niklasson, "Forward-scattering ratios and average pathlength parameter in radiative transfer models," Applied Optics 36, 3735-3738 (1997).

25. C. Tonon, C. Rozé, T. Girasole, and M. Dinguirard, "Four-flux model for a multilayer, plane absorbing and scattering medium: application to the optical degradation of white paint in a space environment." Applied Optics 40, 3718-25 (2001).

26. G. Gouesbet, "Debye Series Formulation for Generalized Lorenz-Mie Theory with the Bromwich Method," Particle \& Particle Systems Characterization 20, 382-386 (2003).

27. R. Li, X. Han, and K. F. Ren, "Generalized Debye series expansion of electromagnetic plane wave scattering by an infinite multilayered cylinder at oblique incidence," Physical Review E - Statistical, Nonlinear, and Soft Matter Physics 79, 1-16 (2009).

28. F. Xu and J. A. Lock, "Debye series for light scattering by a coated nonspherical particle," Physical Review A - Atomic, Molecular, and Optical Physics 81, 1-16 (2010).

29. U. Kreibig and M. Vollmer, Optical Properties of Metal Clusters (Springer, 1995).

30. C. F. Bohren and D. R. Huffman, Absorption and Scattering of Light by Small Particles (Wiley-VCH, Weinheim).

31. L. Simonot, R. D. Hersch, M. Hébert, and S. Mazauric, "Multilayer four-flux matrix model accounting for directional-diffuse light transfers," Applied Optics 55, 27-37 (2016)

32. B. Slovick, Z. Flom, L. Zipp, and S. Krishnamurthy, "Transfer matrix method for four-flux radiative transfer," Applied Optics 56, 5890-5896 (2017).

33. C. Mätzler, "MATLAB Functions for Mie Scattering and Absorption," IAP Research Report pp. 1-18 (2002).

34. J. M. Dlugach, M. I. Mishchenko, L. Liu, and D. W. Mackowski, "Numerically exact computer simulations of light scattering by densely packed, random particulate media," Journal of Quantitative Spectroscopy and Radiative Transfer 112, 2068-2078 (2011).

35. G. A. Niklasson, "Comparison between four flux theory and multiple scattering theory," Applied Optics 26, 4034-4036 (1987). 